CAHIERS DE

NARRATOLOGIE

\section{Cahiers de Narratologie}

Analyse et théorie narratives

$16 \mid 2009$

Images et récits

\title{
Expanded literature. Réflexions autour des « mots du cinéma » dans la prose tabucchienne
}

\section{Stefano Leoncini}

\section{(2) OpenEdition}

Journals

Édition électronique

URL : http://journals.openedition.org/narratologie/968

DOI : 10.4000/narratologie.968

ISSN : 1765-307X

Éditeur

LIRCES

\section{Référence électronique}

Stefano Leoncini, « Expanded literature. Réflexions autour des « mots du cinéma » dans la prose tabucchienne », Cahiers de Narratologie [En ligne], 16 | 2009, mis en ligne le 25 mai 2009, consulté le 15 novembre 2019. URL : http://journals.openedition.org/narratologie/968; DOI : 10.4000/narratologie. 968

Ce document a été généré automatiquement le 15 novembre 2019.

\section{c) (†) $९$}

Cahiers de Narratologie - Analyse et théorie narratives est mis à disposition selon les termes de la licence Creative Commons Attribution - Pas d'Utilisation Commerciale - Pas de Modification 4.0 International. 


\title{
Expanded literature. Réflexions autour des « mots du cinéma » dans la prose tabucchienne
}

\author{
Stefano Leoncini
}

1 S'il est vrai que la convergence entre les moyens d'expression est l'un des traits caractérisants de la post-modernité artistique, Antonio Tabucchi s'impose dans le panorama de la culture italienne et européenne comme l'un des écrivains les plus soucieux d'introduire la littérature dans ce mouvement de confluence des arts, dont la préférence contemporaine pour l'image menace constamment de la laisser à l'écart. L'intellectuel Tabucchi en effet ne considère nullement la chute des frontières de l'art comme un perte mais comme une profonde maturation de ses possibilités, et va jusqu'à préconiser la «fraternité » entre tous les moyens de l'expression artistique ${ }^{1}$. Parmi eux, les arts visuels sont ceux qui le plus jouissent de la faveur du romancier pisan, ceux qui, en vertu de leur nature référentielle, semblent inspirer le plus son écriture.

Une écriture qui développe continuellement des «effets de réel » avec une forte tendance iconique [...] : la figurativisation du référent, qui en est un des aspects dominants - se réalise à travers la constance du descriptivisme analytique, chromatique et morphologique de toutes les formes présentes dans le texte. Que l'on ajoute à cela cet amour tout particulier que Tabucchi voue au «signe analogique " des peintres dont les tableaux deviennent souvent l'objet d'une description [...] le réalisme visuel et l'effet iconique implicite dans de tels procédés, outre à posséder un trait de légèreté, écartent l'opposition traditionnelle entre réalisme et fantastique, à l'avantage d'une forme extatique de la mimesis que Tabucchi semble parfaitement incarner. En cela, si l'on voulait l'enfermer dans une étiquette, ce ne pourrait être que celle, positivement contradictoire, d'un réaliste de la virtualité.$»(\mathrm{tdr})$

2 Cette citation illustre bien pourquoi, dans l'œuvre de restauration de la centralité culturelle du roman entreprise par Tabucchi, le cinéma occupe au sein même des arts iconiques une place privilégiée : c'est parce que, comme l'avait dit Pier Paolo Pasolini, il se fait « avec des bouts de réalité ». Le paradoxe de cette forte connivence que l'écriture tabucchienne entretient avec le septième art n'est pas pour autant expliqué et appelle 
aujourd'hui un questionnement auquel la critique et la recherche peuvent difficilement se soustraire. La présente étude entend prolonger un mouvement amorcé il y a quelques années et repris lors du récent congrès d'Aix-en-Provence, en introduisant expérimentalement un principe de complémentarité disciplinaire entre la critique littéraire et les études cinématographiques.

I. L'intermédialité littérature-cinéma

3 Une alliance objective entre la littérature et le cinéma s'était nouée dès l'aube du siècle, à l'âge où ce dernier, s'étant départi de la vocation strictement scientifique qui l'avait caractérisé à sa naissance, commençait à s'affirmer comme spectacle bourgeois et populaire; puis elle s'était consolidée à l'époque où le long métrage s'imposait comme le format dominant de la production cinématographique. Le film n'a jamais été autant tributaire du roman qu'au moment où il s'émancipe des conventions scéniques de matrice théâtrale et se lance à la conquête de l'Histoire et des espaces naturels. Cette tendance naît en Italie dans le courant des années 10, lorsque les case cinematografiche turinoises, romaines ou milanaises s'engagent dans la production de films de plus en plus riches, longs et narrativement charpentés. Les plus célèbres des kolossal, aujourd'hui appelés avec un bel anachronisme "péplums ${ }^{3}$ ", sont tirés de romans ${ }^{4}$; le premier réalisme transalpin puise, lui, directement son inspiration dans le vérisme et le naturalisme, préfigurant ainsi le paradoxe viscontien, celui d'un (néo)réalisme « médiatisé » par la littérature. Au-delà de la diatribe symbolique (mais de brève durée) sur la primauté et la pureté de l'image, le cinéma n'a jamais hésité à reconnaitre ses dettes envers le roman ou le drame. La réciproque n'est pas vraie, et rares sont les écrivains du XXe siècle ayant admis l'influence que cet art nouveau avait pu exercer sur leur esprit et sur leur appréhension du monde. Italo Calvino, estimateur précoce de Tabucchi, est de ceux-là, qui rédigea son testament de cinéphile sous forme d'une Autobiographie d'un spectateur ${ }^{5}$ où est racontée la longue aventure sentimentale de l'écrivain avec les films, en particulier ceux d'importation hollywoodienne ${ }^{6}$.

I.1 La fonction intertextuelle et la puissance évocatrice du cinéma

4 Chez Tabucchi les mots du cinéma, les références à ses concepts théoriques pullulent, et dans le texte et dans le paratexte, à tel point qu'il convient d'y voir une « fonction intertextuelle » de première importance. Celle-ci peut se définir comme la référence, le renvoi à des « histoires ", à des figures, à des discours extérieurs au récit en cours. Dans les pages tabucchiennes, cette fonction est assez souvent remplie par la citation de titres de films ou de genres cinématographiques bien connus du lecteur potentiel. Dans Nocturne indien, le narrateur présente ainsi l'hôtel Zaouri à Goa :

Des hôtels comme celui-ci peuplent déjà notre imaginaire : nous les avons trouvés dans les romans de Conrad ou de Maugham, dans des films américains tirés des romans de Kipling ou de Bromfield: il nous paraît presque familier » (Notturno indiano, p.80, tdr)

5 Par référence à l'image stéréotypique de l'hôtel colonial véhiculée par la littérature ou le cinéma, l'auteur fait l'économie de la description, lui préférant le raccourci visuel, de par sa nature très riche d'harmoniques tonales et chromatiques. Parfois l'intertextualité littérature-cinéma devient transitive ou "à double ressort ». Lorsque le personnage d'un récit, s'adressant à son interlocuteur, fait allusion au roman de Kafka Le Procès et aussitôt après évoque un film qui en est l'adaptation cinématographique (The trial d'Orson Welles, 1962) la référence s'intériorise à la diégèse. Il n'y a pas de hiérarchie à établir entre les deux arts, ni de frontière à marquer entre une culture "élitaire " (la littérature) et une culture "populaire » (le cinéma), 
selon une définition bien connue du postmodernisme ${ }^{7}$. Le but avoué est ici de « croiser l'information" afin de conjurer l'incertitude de la communication. Cette fonction première et apparente se double toutefois d'un effet plus discret de résonance sur le « lecteur modèle » de Tabucchi. Le Fil de l'horizon contient une variante ultérieure de ce qu'on pourrait définir la "référence transitive »: Spino, employé de la morgue de Gênes, mène une investigation sur l'existence d'un défunt, en se livrant à une expérience fort semblable à celle qu'entreprend le photographe Thomas dans Blow up (Michelangelo Antonioni, 1966). Comme ce dernier, il traque les indices d'une vie sur de vielles photos qu'il élabore à l'infini, les "gonflant » afin de l'étudier dans les plus petits détails, et jusqu'à se rendre compte qu'elle ne peuvent lui délivrer aucun enseignement concernant l'être humain sinon qu'il demeure irréductiblement mystérieux. Le film d'Antonioni est librement inspiré de Las babas del diablo de Julio Cortázar, une référence "érudite» qui n'est probablement pas contenue dans l' «encyclopédie personnelle» du lecteur moyen, et à laquelle celui-ci ne pourra remonter sinon au prix d'une démarche volontaire d'information. C'est précisément en cela que consiste la dynamique implicite de l'écriture tabucchienne : en le désir - perçu comme nécessité - d'entretenir chez le lecteur la curiosité culturelle. On se contentera pour l'instant de cette simple remarque, que la citation n'a jamais chez Tabucchi l'effet d'une "clôture » ésotérique, élitaire, du texte, mais tout au contraire l'effet d'un "volant», d'une extension qui garantit au récit un souffle plus long, éventuellement amplifié par le bagage culturel (actuel ou potentiel) du lecteur.

6 Parmi tous les textes de Tabucchi, celui qui contient la plus forte dimension intertextuelle est sans doute Forbidden Games, présent dans le recueil Il se fait de plus en plus tard. Dans un tissu presque inextricable de références (littéraires, musicales, linguistiques, culturelles au sens large), ce récit dont le titre fait allusion à Jeux interdits de René Clément ne cite pourtant que deux films. Qui plus est - suivant un goût du paradoxe et de la contradiction souvent rencontrés chez notre auteur - le scripteur y conclame l'intertextualité comme un phénomène appartenant à un âge révolu!

Je rentrais à pied, souvent très tard la nuit, car le métro fermait assez tôt et je restais regarder des films de cinéclub dans un petit cinéma de Saint-Germain : L'Age d'or, Un Chien andalou. Des choses comme ça. Je croyais aux avant-gardes. C'était beau de penser qu'elles étaient révolutionnaires. Esthétiquement révolutionnaires, s'entend. (SSFSPT, p.42)

Ici, le caractère privé, voire hermétique des propos du « je » narrateur s'accorde mal à la dimension collective, sociale, d'ouverture, que l'on reconnaît au cinéma. Beaucoup plus souvent, la culture - l'hypertexte - cinématographique devient terrain de discussion, d'entente ou de complicité entre les personnages du récit ${ }^{8}$. Dans ce contexte dialogique, un titre de film est un "raccourci» vers une idée, un sentiment, une atmosphère. Chacun peut en faire l'expérience dans la conversation courante. La préférence pour la référence cinématographique doit être considérée comme un expédient narratif de la concision dont Tabucchi est un fervent adepte, voire du laconisme qui sert si bien sa stratégie poétique; plus on évoque, en effet, moins on a à décrire, à raconter. En même temps, le moins-disant est une preuve de la confiance que l'auteur repose en son lecteur, en sa capacité à participer à la construction du sens, tâche qu'il lui délègue volontiers. Côté création, la dette envers la culture cinématographique est aisément reconnue. Dans la note introductive à Petites équivoques sans importance, où il fait explicitement référence à Calderón et à l'onirisme baroque, Tabucchi explique la genèse de la nouvelle Cinema, en révélant qu'elle « doit 
beaucoup à une soirée de pluie, à une petite gare de la Riviera ligure et au visage d'une actrice disparue »: des clichés étroitement liées à l'imaginaire cinématographique. L'effet escompté sur l'esprit du lecteur est symétrique à celui qu'il arrive à l'écrivain d'éprouver lui-même en tant que spectateur de cinéma : il l'introduit à la rêverie.

I. 2 Le cinéma, étape du parcours onirique

Le paradigme onirique tabucchien comporte une variété de manifestations qui s'étend de l'irréel de la fable populaire au rêve éveillé et jusqu'au cauchemar, en passant par l'hallucination. Les contours de chaque étape du parcours ne sont pas nettement définis : au contraire, l'écrivain se plait à les brouiller. Requiem, texte suspendu entre deux régimes proches mais distincts (l'irréel et l'onirique, certains de ses chapitres se déclarant comme des rêves) se présente au public comme Uma halucinaçao ${ }^{9}$, incertitude recherchée qui ne frappe pas seulement le commun des lecteurs: Alain Tanner, qui adapte le roman pour le grand écran en 1998, révèle les scrupules stylistiques qui ont été les siens quant à la modalisation visuelle à donner à chaque épisode dans cette histoire de deuils symboliques. La difficulté est résolue par la mise en œuvre d'un travail particulier sur la temporalité, qui a pour effet le maintien de toute l'ambiguïté perçue dans le livre.

On craignait que le spectateur prenne toute l'histoire pour un rêve. Pour Tabucchi, c'est une hallucination et pour moi qui n'ai jamais vécu d'hallucination ni rencontré de fantôme de ma vie, j'ai voulu croire à une réalité irréelle. Mais le plus important reste que le temps est disloqué, le temps linéaire n'existe plus, ou bien est en boucle $^{10}$.

9 Le cinéaste lausannois soulignait à la même occasion la "cinématographicité " intrinsèque $d u$ récit tabucchien, qui d'après lui ne présentait aucun motif d'infidélité dans son adaptation filmique ${ }^{11}$. Pour l'écrivain et pour ses personnages, le rêve assure des fonctions de simplification, d'unification du réel - parfois même de « correction du définitif ", glose Tabucchi. Il communique avec le souvenir et la mémoire, dans le contexte d'une stratégie consolatoire que la psyché se construit elle-même dans un but d'autodéfense.

Dans le rêve, la circonstance acquiert un caractère vague qui améliore l'image. La réalité passée est toujours moins pire [meno peggio] que ce qui fut réellement: la mémoire est un formidable faussaire. On fait des contaminations, sans même le vouloir.

Les paradis artificiels s'inscrivent eux aussi au nombre de ces « états seconds » du corps et de l'esprit, qui permettent à l'homme de fuir la tyrannie du réel: en particulier, l'ivresse et le saper bere, considérés comme des marques de civilisation ${ }^{12}$. Autre idée qui revient avec fréquence, les rêves servent à boucher les trous, à combler les lacunes laissées par la rationalité. Chère aux romantiques, qui n'ont pas réellement connu le cinéma, elle est reprise par les décadents, eux parfaitement conscients de ses potentialités : et, si la référence à D'Annunzio n'est pas de mise (il est, avec Marinetti, l'un des "écrivains-repoussoirs », comme les a appelés la critique tabucchienne), le renvoi à Pirandello (en revanche un de ses " écrivains-miroir ») est inévitable, qui dès 1924 avait perçu dans l'image filmique la possibilité d'outrepasser les limites du verbe en termes d'expression du rêve, du souvenir, de l'hallucination, de la folie, du dédoublement de la personnalité. L'écrivain sicilien avait déclaré reposer de grands espoirs en les moyens plastiques du cinéma, et appelé les " cinématographistes » à un effort créatif ${ }^{13}$. La réponse des cinéastes dans l'exploration d'un au-delà du récit a été à la hauteur des attentes pirandelliennes. Le répertoire visuel de l'onirique engendré tout 
au long du siècle alimente largement la représentation du rêve que l'homme occidental se fait aujourd'hui et sa richesse expressive exploitée par les écrivains modernes. Que Tabucchi décrive souvent ses rêves par référence au cinéma n'exige guère d'être démontré : voici néanmoins un exemple particulièrement significatif, tiré du récit Storia di una storia che non c'è dans lequel il est question d'une histoire longtemps rêvée et jamais écrite :

[Cette histoire], je la vois en noir et blanc, c'est ainsi que je rêve habituellement; ou bien en couleurs estompées et très pâles; le tout baignant dans une brume légère, un voile qui en atténue et en émousse les formes. L'écran sur lequel elle est projetée est le ciel nocturne d'un littoral atlantique ${ }^{14}$, devant une vieille maison qui s'appelait São José da Guia (I volatili di Beato Angelico, p.61, tdr)

11 Ce goût typiquement cinématographique pour le jeu sur la contiguïté des niveaux de réalité s'était manifesté dès le premier roman, Piazza d'Italia, où Don Milvio, curé aux tendances marxistes, «se fait un film » sur sa " machine hydraulique de l'égalité » sous l'effet de la somnolence postprandiale. Le fonctionnement et les bénéfices de l'appareil, que son concepteur voit en rêve, sont décrits à l'indicatif présent, tandis que rien ne vient marquer linguistiquement dans le texte le passage du "rêve éveillé » au sommeil dans sa phase onirique (Piazza d'Italia p. 44-45). Dans les lignes suivantes, dont on attend qu'elles lèvent l'ambiguïté pour le lecteur, Tabucchi résout dans le langage l'antithèse rêve/réalité. Il le fait avec un art du compromis qui lui est typique, en parlant de la «vision » de Don Milvio. Au fils des pages et des livres, il finit par s'instaurer entre le rêve et l'image cinématographique un principe d'équivalence, une forme d'homogénéité, qui permet à certains éléments de passer plus ou moins subrepticement d'un ensemble à l'autre. Dans le chapitre 8 de Nocturne indien, lorsque le « je » narrateur se rend à Goa, éclat d'Occident incarné dans le subcontinent indien, il visite l'archevêché de $\mathrm{S}$. Boaventura où il se trouve plongé dans un état de contemplation; glissant impromptu dans le sommeil, il rêve d'Alphonse le Grand ${ }^{15}$, qui lui apparait sous les dépouilles de l'acteur Nicolaï Tcherkassov, interprète du Tsar dans le film d'Eisenstein Ivan le Terrible (1944). Italo Calvino, « collectionneur de stars et comparses ${ }^{16}$ ", nous avait fourni une attestation précoce de l'empreinte que la figure actoriale peut laisser sur le specateur-écrivain. Tabucchi, quelques années après l'adaptation cinématographique de Sostiene Pereira, révèlera que depuis sa vision du film le personnage du journaliste lisboète, qui n'est pas décrit physiquement dans le roman mais qui possédait dans l'esprit de l'écrivain une physionomie parfaitement définie, a désormais pour lui les traits du Pereira incarné par Marcello Mastroianni ${ }^{17}$.

II La référence aux procédés narratifs cinématographiques

12 La contiguïté entre l'écriture de Tabucchi et la création filmique ne se limite évidemment pas à la présence de mots, noms, visages et atmosphères liés au cinéma. Une «homologie structurelle", selon les termes employés par Hector Freire à propos de la nouvelle Cinema $^{18}$, se profile dès le premier roman publié. Piazza d'Italia, sorte de contre-histoire de l'Italie contemporaine racontée du point de vue des vaincus, s'ouvre par un épilogue, catégorie purement littéraire qui toutefois nous fait percevoir la suite du récit comme un flash-back, et l'ensemble de sa structure narrative comme étant d'emprunt cinématographique. Le roman s'articule en 3 parties, appelées tempi ${ }^{19}$, évoquant certaines grandes fresques historiques du cinéma italien ${ }^{20}$. Chaque partie se subdivise à son tour en de très brefs chapitres, d'une longueur moyenne à peine supérieure à une page. Vingt ans après la sortie de Piazza d'Italia, Antonio Tabucchi se confiera à Antoine de Baecque dans le cadre d'un numéro spécial des Cahiers du cinéma, 
intitulé Le cinéma des écrivains, en expliquant la construction narrative si particulière du roman par la découverte des Leçons de cinéma de Sergueï Eisenstein et, par ailleurs, en exprimant le sentiment d'une "fraternité d'inspiration» avec Theo Anghelopoulos, cinéaste du Voyage des comédiens. Si l'explication que Tabucchi donne lui-même de la genèse de Piazza d'Italia semble peu convaincante, les extrapolations qu'en a fait la critique, reprenant un peu naïvement à son compte les «propos » de l'auteur, le sont encore moins. Pour commencer, et si l'on s'en tient rigoureusement au plan des mots, les «micro-chapitres" du livre ne peuvent en aucune manière être comparés à des photogrammes, ni même à des plans : sous leur aspect syntagmatique (et du point de vue du contenu narratif), ils sont plutôt à considérer comme l'équivalent de séquences. Mais, d'une façon plus générale, l'impression qui se dégage des déclarations de Tabucchi quant à sa découverte des théories eisensteiniennes est que leur impact a été " poétique » bien plus que technique; en ce sens que le concept de la " productivité du montage » développé par le cinéaste russe a vraisemblablement conforté l'écrivain italien dans son inclination « naturelle » à composer par fragments. « Je me suis résigné à écrire selon ma nature ${ }^{21}$, confiera-t-il beaucoup plus tard dans le prologue au receuil Femme de Porto Pim.

II.1 Les avatars de la dialectique simple/multiple : montage parallèle, surimpression

L'œuvre de Tabucchi se présente ainsi au lecteur comme un "tout fragmentaire", concept paradoxal s'il en est mais seul à même de rendre l'idée qu'ici chaque élément ne vaut pas tant pour lui-même que dans son rapport aux autres, et ce quelque soit le niveau textuel où l'on veuille se situer. Nombre de récits ou de pages apparemment anodins dans leur hyponarrativité caractéristique acquièrent tout leur sens une fois insérés dans le macrotexte constitué par l'opera omnia. En ce sens, le « lecteur modèle ${ }^{22}$ » de Tabucchi s'apparente au cinéphile, il est semblable à ce spectateur qui, par affinité avec tel "auteur", s'accomode de la difficulté d'approche de son "écriture ", à condition qu'on lui en livre quelques clés de lecture. Chez Tabucchi comme chez la plupart des «auteurs» cinématographiques, le plaisir esthétique et le travail herméneutique appellent une attention et une fréquentation qui attirent le destinataire hors des territoires de la "consommation » courante : les clés de lecture se décèlent dans et à travers la répétition. Dans l'oeuvre de Tabucchi comme dans celle d'autres artistes plus ou moins énigmatiques ${ }^{23}$, la jouissance de l'œuvre participe d'une quête, d'un jeu de pistes ou, si l'on veut, d'une chasse au trésor où le trésor se recèle dans le plaisir même de la recherche. Mais cette homologie entre l'œuvre de Tabucchi et le cinéma ne se borne pas à la similitude entre sa manière de concevoir l'écriture et les avatars du « film d'art ». Elle se manifeste dans leur nature profonde, l'une et l'autre se fondant sur une dialectique unité-multiplicité. L'écriture de Tabucchi obéit à une logique compositive du fragment, mais aussi à celle du jeu de puzzle, où chaque pièce, pour faire sens, doit être agencée avec toutes les autres; le cinéma procède lui d'un " constant passage [...] de l'un au multiple puis du multiple à l'un ", les photogrammes, "malgré leur caractère composite et multiple, [ayant] comme fonction de donner l'illusion d'une unité et d'une continuité sans faille». Le paradoxe "que les images animées soient faites d'images arrêtées » ne s'arrête pas là : « le mouvement dialectique de la contradiction "simple/multiple" connait d'autres ordres de manifestation, s'agissant du cinéma, par la procédure du montage ${ }^{24}$.» Le dernier micro-chapitre de Piazza d'Italia est assez significatif de ce qui semble être pour Tabucchi une forte exigence poétique et expressive. 
15. La mort ne s'achète pas

(deux tableaux en un, pour le fait d'être simultanés)

$(\mathrm{tdr})$ de chapitre. Deux actions de l'histoire, censées se dérouler en même temps, y sont racontées par récits alternés finissant ensuite pas se conjoindre rejoignant chronologiquement la première "image » du livre, celle où l'on "voit " Garibaldo frappé au front par une balle de fusil. La critique a rapproché cette construction narrative de ce que l'on appelle au cinéma le «montage en parallèle ${ }^{25}$ ", construction qui seule serait capable de rendre la simultanéité de deux actions éloignées dans le temps. En réalité, le montage en parallèle n'imite pas mieux la simultanéité que ne le fait l'écriture, puisqu'il procède lui aussi par alternance de vues. Le seul procédé écranique permettant la monstration simultanée de deux actions est en fait le split screen ou écran partagé; or, une telle bipartition de la surface signifiante n'est pas autorisée par la forme écrite, du moins dans la forme conventionnelle du genre « roman ». Quant à la technique de la surimpression, au cinéma elle est généralement employée à d'autres fins expressives, comme la visualisation d'images mentales. Elle permet par ailleurs les fondus-enchainés, ainsi que les effets de morphage/morphing (avant que l'informatique ne s'en empare). On observe quelque chose d'apparenté à ce type d'effet spécial dans cet extrait, où le scripteur s'adresse en rêve à son père défunt :

C'est étrange comme il peut advenir parfois de superposer deux souvenirs en un seul souvenir, c'est ce qui était en train de m'arriver, je me rappelais ton image de mille neuf cent cinquante-six et j'en faisais un amalgame avec l'image que tu m'aurais laissée de toi, trente ans plus tard ("Gli archivi di Macao », in I volatili di Beato Angelico, p. 73-74, tdr).

Cet écrasement de la mémoire et, à travers elle, du temps, est une figure très fréquente chez notre écrivain et semble faire appel à l'expérience visuelle que les lecteurs peuvent avoir des effets ciné- ou vidéographiques.

\section{II.2 Déconstruction et réalisme : analepse, métalepse, pirandellisme}

Le roman Piazza d'Italia est écrit à l'époque où le mot d'ordre chez les cinéastes européens était la "déconstruction », celle où les films étaient montés «contre » la façon dont ils étaient tournés. Enjeu idéologique s'il en est, il consistait d'une part à montrer l'artificialité du "cinéma de la transparence », le mensonge de ce camouflage du travail de production au profit d'une naturalité apparente ; mais aussi, en dernière instance, à tenter d'instaurer un nouveau rapport du public à l'image et - à travers celui-ci - un nouveau rapport à la réalitée ${ }^{6}$. Dans Piazza d'Italia, l' «épilogue ", placé en ouverture, installe d'emblée le principe du raccourci temporel. En même temps, il ébauche ce mouvement d'aller et de retour constant dans le temps historique qui caractérise le "montage » temporel du roman, de l'Unité d'Italie à la Libération et à l'après-guerre, le tout en trois pages et trois micro-chapitres, selon le schéma ici reproduit sous forme de tableau.

\begin{tabular}{|l|l|l|l|}
\hline Epilogue - « S’è sciolto il fiocco » & T 0 & Mort de Garibaldo- Volturno & 1948 \\
\hline \hline 1. « C'è ancora un po' di tempo » & T-1 & Mort de Garibaldo-Plinio & $188 ?$ \\
\hline \hline 2. « Si cambia padrone » & T-2 & Remplacement de la statue & 1865 \\
\hline
\end{tabular}


17 Par la suite, le récit enchaîne les avancées et les retours dans le temps, à tel point que le lecteur est parfois contraint de revenir en arrière dans les pages pour comprendre la concaténation des faits ${ }^{27}$. Ce travail d'élaboration de la temporalité trouve d'étonnants échos dans le cinéma : Piazza d'Italia est publié entre l'année de sortie en salle de Drame de la jalousie (1970) et celle de Nous nous sommes tant aimés (1975), d'Ettore Scola, cinéaste reconnu comme l'un des tout premiers à avoir introduit des procédés d'enchâssement dans des films destinés au plus vaste public. La métalepse narrative, ou franchissement de frontière entre récit premier et récit enchâssé, est une transgression de l'une des règles de base du cinéma classique, règle qui vise à "préserver la confiance dans l'écran ", selon les mots de Iouri Lotman. Chez Tabucchi, l' " analepse problématique ", (appelée "pirandellisme» par André Bazin), fonctionne principalement au niveau intertextuel, lorsque des récits distincts représentent les mêmes personnages et font références aux mêmes situations, parfois à quelques années de distance. L'exemple le plus significatif dans l'œuvre de notre écrivain est celui qui voit se rapprocher - jusqu'à l'impression fallacieuse d'unité - l'univers diégétique du chapitre VI de Nocturne indien et celui qu'évoque la lettre de Monsieur Janata Monroy, contenue dans le recueil Les Oiseaux de Fra Angelico (I volatili del Beato Angelico, 1987). Tout en se renvoyant l'un à l'autre, ces textes se contredisent mutuellement de façon patente ${ }^{28}$ : toutefois, pour déceler leur incompatibilité en logique, il est nécessaire de les connaître tous les deux car bien sûr chacun se tient parfaitement pris dans sa singularité. Cette forme de relativisme souvent professé par Tabucchi prend ailleurs les formes de la dialectique, bien connue par les filmologues, entre cadre et hors-cadre, champ et hors-champ.

II.3 Propriétés iconiques dans le texte : hors-cadre et hors-champ

Selon la théorie cinématographique, le hors-cadre se définit le plus souvent comme cet «espace de la production filmique où se déploie et où joue tout l'appareillage technique, tout le travail de réalisation, et métaphoriquement tout le travail d'écriture ${ }^{29}$ ". Dans le cinéma classique, il est même ce qui nous permet de désigner da la façon la plus sûre l'instance narrative, qui est « elle-même ce qui généralement reste hors-cadre » (id. p. 79). Enfin, il est le lieu où le dispositif cinématographique se cache à la (fausse-) conscience spectatorielle. C'est en cela qu'il est rapprochable de la notion de «morceaux choisis ", qui aura une grande fortune auprès de la critique et sera reprise par Tabucchi lui-même dans d'autres textes. Dans le denier chapitre de Nocturne indien, le protagoniste rencontre Christine, une jeune femme francophone qui se définit "photographe d'abjection». Celle-ci lui raconte sa plus belle réalisation: un livre reproduisant une série de photos prises en Afrique. Sur la première photo, qui est un agrandissement, on voit un jeune noir levant les bras au ciel, le visage crispé comme par une effort physique victorieux. La première photo est soulignée par une didascalie, la seule du livre: "Méfiez-vous des morceaux choisis ». Bien que le mot n'apparaisse que dans l'épilogue, l'idée était déjà énoncée au chapitre I du roman.

Je [connaissais] le "Quartier des Cages» à travers les photographies d'un photographe célèbre et je croyais être préparé à la misère humaine, mais les photographies renferment le visible dans un rectangle. Le visible sans cadre (senza cornice), c'est toujours autre chose (Notturno indiano, p.15, tdr)

19 Dans le dernier chapitre encore le personnage de Christine fera allusion à l'effet " cache» du cadre, qu'il soit l'élément réel et concret qui détermine les bords de l'image photographique et cinématographique ou qu'il soit métaphoriquement entendu. "Morceaux choisis » prend donc ici le sens négatif, péjoratif, de "vérité partielle », de falsification ou d'édulcoration de la réalité. A la fin du chapitre, le 
protagoniste reprend la formule à son compte et, en vertu de la forte dimension métanarrative du passage, affirme à son tour : «l'agrandissement fausse le contexte, il faut voir les choses de loin ». Le sens global du roman, toutefois, nous dispense une autre sagesse : il sous-entend en effet que la bonne posture consiste non pas à prendre de la distance, du recul, mais plutôt à se considérer comme faisant partie du champ, tout en gardant un regard extérieur sur soi-même. Un point de vue cher à Tabucchi et une belle définition de ce en quoi consiste et de ce que nous permet, virtuellement, la spectatorialité cinématographique.

Le hors-champ, souvent confondu avec le hors-cadre, est en réalité d'une tout autre nature: il est dans la terminologie des études cinématographiques la prolongation imaginaire des éléments du champ dans un univers homogène, que le spectateur construit à partir de celui-ci. Si donc le hors-cadre est hétérogène au visible, le horschamp lui est homogène et n'existe que par rapport à lui. Le champ stimule ainsi chez l'instance spectatorielle la capacité d'inférence, l'appelant à créer « autour » du visible, c'est-à-dire autour du champ, tout cet univers que l'image ne peut pas montrer, d'une part parce qu'elle n'est pas illimitée, d'autre part parque la représentation cinématographique est, le plus souvent, construction. L'écriture tabucchienne fonctionne elle aussi par de continuels appels à l'inférence, notamment intertextuelle. Bien qu'il lui arrive souvent de critiquer la logique, il ne cesse de solliciter le lecteur à un travail de mise en relation. Le roman-puzzle Piazza d'Italia n'en est que le premier exemple: la préférence marquée d'Antonio Tabucchi pour la forme fragmentaire du « recueil » de textes brefs, récits, nouvelles, rêves, lettres, ne fera que se confirmer et, par moments ou par endroits, s'accroître au fil de sa carrière littéraire.

\section{BIBLIOGRAPHIE}

Antonio Tabucchi: a Collection of Essays, in "Spunti e ricerche" (sld) Bruno Ferraro et Nicole Prunster, vol. 12 (1996-97)

AUMONT Jacques - BERGALA Alain - MARIE Michel - VERNET Marc, Esthétique du film, Nathan Université, Paris, 1988.

BOSCHI, Carina, « Fruizione tematica e funzioni poetiche del cinema nella narrativa di Antonio Tabucchi », in Echi di Tabucchi, cf. infra.

CALVINO, Italo, « Autobiographie d'un spectateur », in F. Fellini, Faire un film, Seuil, 1996.

CESERANI, Remo, “The art of fixing shadows and writing with light", in Antonio Tabucchi: a Collection of Essays, cf. supra.

COSTA Antonio, Nel corpo della parola, l'immagine : quando la letteratura cita il cinema, «Contemporanea », 3, 2005.

Echi di Tabucchi / Echos de Tabucchi, Actes du colloque international d'Aix-en-Provence, 12-13 janvier 2007, Revue d'études italiennes, 2007.

FERRO, Roberto (sld), Sostiene Tabucchi, Buenos Aires, Editorial Biblos, 1999. 
REIRE, Hector, “Todo es una película”, in R. Ferro (sld), Sostiene Tabucchi, cf. supra.

GAUDREAULT, André, Du simple au multiple : le cinéma comme série de séries, in «Cinémas » Volume 13, numéros 1-2, Automne 2002 (« Limite(s) du montage »),

PALMIERI, Giovanni, Antonio “Tabucchi's Iconic Temptations”, in Antonio Tabucchi: a Collection of Essays, cf. supra.

RIMINI, Thea, « La (cine)biblioteca di Tabucchi : il montaggio di Piazza d'Italia », in Echi di Tabucchi, cf. supra.

\section{NOTES}

1. Conversazione con Antonio Tabucchi. Dove va il romanzo, P. Gaglianone et M. Cassini (sld), Roma, Il Libro che non c'è, 1995, p. 12.

2. G. Palmieri, "Una volatile leggerezza : il « lato manco » di Antonio Tabucchi”, in Nathlie Roelens et Inge Lanslots, Piccoli equivoci con importanza, Actes du colloque d'Anvers, 1994, p.123-131.

3. Le terme a été forgé par la critique française des années 50 à propos du film en costume d'après-guerre. La critique et l'historiographie cinématographique italienne l'ont adopté plus récemment.

4. Les Derniers Jours de Pompéi est tiré d'un roman victorien; Quo Vadis est un roman polonais d'abord publié sous forme de feuilleton ; Cabiria, " frauduleusement » attribué à Gabriele D'Annunzio dans un but de marketing, s'inspire largement de Cartagine in fiamme d'Emilio Salgari.

5. I. Calvino, « Autobiographie d'un spectateur », in Faire un film, Seuil, 1996. La préférence pour le cinéma hollywoodien est sans doute à mettre au compte de cette nostalgie pour une « grande structure » souvent professée par Tabucchi. Calvino regrette la disparition, après-guerre, du « cinéma des majordomes » (op. cit. p.23). Ce n'est sans doute pas un hasard si des figures inoubliables de majordomes, de garçons ou de barmen élégants et courtois apparaissent dans Damasceno Monteiro, Requiem, Sostiene Pereira.

6. Selon Calvino, les films italiens ne peuvent, eux, passer dans ce discours du cinéma comme « autre dimension du monde » (Calvino, ibid. p.22), précepte qui s'applique également fort bien à l'écriture de Tabucchi, toujours en quête d' " ailleurs » géographiques et métaphoriques.

7. "The effacement [...] of the older (essentially high-modernist) frontier between high culture and so-called mass or commercial culture". Cf. Fredric Jameson Postmodernism, or, The Cultural Logic of Late Capitalism, Verso, 1991. La distinction est moins pertinente en Europe, où le cinéma aspire au statut d'art dès ses toutes premières expressions. 8. Cet aspect est approfondi par Carina Boschi, cf. « Fruizione tematica e funzioni poetiche del cinema nella narrativa di Antonio Tabucchi ", in Echi di Tabucchi/Echos de Tabucchi, Actes du congrès d'Aix-en-Provence, p. 349-373.

9. C'est le sous-titre de roman, écrit en portugais.

10. L'Humanité - Cultures, 3 juin 1998.

11. « On est resté fidèle à son récit parce qu'on n'avait aucune raison d'être infidèle », Ibid. Une affirmation qui semble contredite par celle du cinéaste portugais Fernando Lopes, qui adapta en 1993 Le fil de l'horizon et qui considère, lui, l'écriture de Tabucchi comme anti-cinématographique. Il faut voir cependant de quel cinéma on parle. Les 
atmosphères oniriques de textes comme Requiem ou La Tête perdue de Damasceno Monteiro, pour ne citer que ceux-là, entrent puissamment en résonnance avec celles que campent les films de João César Monteiro, un auteur iconoclaste, assurément très éloigné du classicisme cinématographique.

12. «Vous savez, quand on a un peu trop bu, la réalité se simplifie, on saute les vides entre les choses, tout semble coller et on dit : j'y suis. Comme dans les rêves. » (tdr) Cf. « Rebus » in Piccoli equivoci senza importanza, p.46; cf. aussi p.29.

13. « C'est un film russe, Le Père Serge, qui pendant la guerre m'a laissé entrevoir les possibilités de cet art jeune : le Rêve, le Souvenir, l'Hallucination, la Folie, le Dédoublement de la personnalité ! [...] Si les cinématographistes le voulaient, il y aurait de grandes choses à faire! », in « Nouvelles Littéraires”, 19 novembre 1924. Yakov Protazanov, Le Père Serge/Otec Sergej, 1917, tiré d'un roman de Léon Tolstoï et interprété par Ivan Mouuzjoukine, qui fut Le feu Mathias Pascal dans la première adaptation filmique de Marcel L'Herbier en 1926. On peut rappeler que Mouzjoukine fut également l'acteur qui prêta son visage aux légendaires expérimentations de Lev Koulechov visant à démontrer « la puissance évocatrice du montage ».

14. Tabucchi voit dans la culture portugaise, qu'il considère comme sa culture d'adoption, l'horizon européen de la rêverie : rêverie exotique (le Brésil, le lointain Orient), historique (l'Empire) et poétique (le fado, la saudade), la terre lusitanienne est l'espace humain où son propre tempérament mélancolique trouve un prolongement idéal chez ses semblables.

15. Afonso de Albuquerque (1453 - 16 décembre 1515, Goa), navigateur et explorateur portugais.

16. I. Calvino, op. cit. p. 23.

17. A propos de la version filmique de Sostiene Pereira, cf. Vito Zagarrio, Cinema italiano anni novanta, Marsilio, Venezia, 1998, p. 92-93.

18. Cf. Hector Freire "Todo es una película", in R. Ferro (sld), Sostiene Tabucchi, Buenos Aires, Editorial Biblos, 1999.

19. Ce mot est à forte consonnance cinématographique. En Italie, jusqu'à un passé relativement récent, les projections en salle étaient coupées en primo tempo, secondo tempo et éventuellement terzo tempo pour les très longs métrages. Ces césures ne relevaient nullement des auteurs mais des distributeurs et donnaient lieu à un bref entracte. Dans certaines salles (Calvino en fait mention, cf. op. cit. p. 16), un toit ouvrant mettait en communication le noir de la salle et celui de la nuit extérieure, créant une sorte d'osmose entre deux ordres de réalité.

20. On pense en particulier à la fresque en deux parties Dix neuf cent de Bernardo Bertolucci (Novecento, 1976), qui, notons-le, est postérieure a Piazza d'Italia. On ne peut ignorer par ailleurs une certaine fraternité d'inspiration avec les cinéastes Paolo et Vittorio Taviani, toscans comme Antonio Tabucchi.

21. « Mi sono rassegnato a scrivere secondo la mia indole ». Cf. Prologue di Donna di Porto Pim, p.9.

22. Les concepts d' " auteur modèle » et de « lecteur modèle » sont développés par Umberto Eco in Lector in fabula, Milan, Bompiani, 1979.

23. Parmi les titres de textes tabucchiens faisant référence aux jeux d'énigmes, « Rébus », « Jeu du revers ».

24. Gaudréault, André, Du simple au multiple : le cinéma comme série de séries, in « Cinémas » Volume 13, numéros 1-2, Automne 2002 («Limite(s) du montage »), «</ span>http://www.erudit.org/revue/cine/2002/v13/n1/007955ar.html>. 
25. Selon la classification des profils séquentiels établie par Christian Metz.

26. « C'est [...] en partie parce qu'elle oscille entre un statut plein de représentation (représenter quelque chose de façon réaliste) et l'extrême évanescence de son matériau (des ombres et des ondes) que l'image de cinéma fascine et retient. Elle requiert du spectateur qu'il ne soit pas un simple témoin, mais aussi quelqu'un qui invoque très fortement le représenté parce qu'il est convaincu du peu de consistance de la représentation ». Cf. Cf. Aumont/Bergala/Marie/Vernet Esthétique du film, p. 105-109. 27. Comme le note Thea Rimini in « La (cine)biblioteca di Tabucchi : il montaggio di Piazza d'Italia », in Actes du congrès d'Aix-en-Provence, op. cit., p. 321-348.

28. Significativement, le deuxième en ordre de parution parmi ces textes s'intitule « La phrase qui suit est fausse. La phrase qui précède est vraie ».

29. Aumont/Bergala/Marie/Vernet, op. cit., p. 19.

\section{INDEX}

Mots-clés : littérature, études cinématographiques, post-moderne, narratologie, intertextualité

\section{AUTEUR \\ STEFANO LEONCINI}

Université de Nice, CIRCPLES 